\title{
Semantic-driven Manufacturing Process Management Automation
}

\author{
Francisco Maciá-Pérez, Virgilio Gilart-Iglesias, Antonio Ferrandiz-Colmeiro, \\ José Vicente Berná-Martínez, Jorge Gea-Martínez. \\ Computer Science and Technology Department, University of Alicante \\ \{pmacia, vgilart, aferrandiz, jvberna, jgea\}@dtic.ua.es
}

\begin{abstract}
The maturity of IT, in conjunction with certain modern theories about Business Processes Management (BPM), has propitiated an appropriate scenario for meeting the needs of new business models, which are increasingly centred on mass customization. Integration solutions provided within the framework of manufacturing organisations have opened up a wide range of possibilities in process management, which, paradoxically, is causing the traditional bottleneck between the enterprise level and production levels to now be transferred to the process management and modelling levels. This paper proposes the incorporation of knowledge into the definition of the processes and services involved by means of an ontology, thus maximising the automation of process modelling and facilitating the work of process engineers.
\end{abstract}

\section{Introduction}

The capacity that the Internet has provided to customers to select the consumer articles that best suit their needs at any given time and at the best prices is causing manufacturing organisations to move from traditional production paradigms centred on mass production to models that facilitate mass customization [1]. This new scenario demands that organisations implement new, more dynamic and more flexible management models that easily enable them to adapt to environmental changes brought about by market demand [2][3].

In order to respond to the needs of new models, the BPM (Business Process Management) paradigm has become the focus of more appropriate business process management [3] because it contemplates continuous change as one of its main characteristics in addition to the dynamic adaptation of the business processes and IT infrastructures that sustain them. BPMS (Business Process Management System) platforms are presented as the IT solution that supports the life cycle of the BPM by facilitating the integration and automation of business processes management in accordance with the requirements of new production models.
Within the framework of manufacturing organisations it is difficult to completely implement BPMSs due to the conceptual and technological gap between the enterprise level and productions levels, caused by the rigidity of the elements located in the lower levels of production. Offering a vision of Industrial Machinery as a Service (IMaaS) [4], integrated in accordance with a ServiceOriented Architecture (SOA) paradigm, has led to a successful proposal for solving this problem by considering industrial machinery manufacturing processes as just another element of the business process management system.

By removing this gap it is possible to dynamically align the business and manufacturing processes with the strategic objectives of the organisation, generated by continuous changes in the market. However, this new scenario is causing the traditional bottleneck between the enterprise level and production levels to now be transferred to the domain of modelling and the composition of processes by significantly increasing the workload of process engineers.

In order to mitigate this workload, the focus proposed in this article consists of the incorporation of knowledge into the definition of the processes and services involved by means of the use of ontologies, thus obtaining a method that enables the composition and modelling of business processes to be automated in accordance with the strategic objectives of the organisation. This enables the excessive workload of process engineers to be reduced.

In order to develop the proposal, the following section presents a review of the state-of-the-art of related aspects. This is followed by the proposal of an ontology that establishes the conceptualisation of the manufacturing processes by facilitating automation within their modelling and composition and a description of the general proposal for a scenario in which industrial machinery is offered as a service (IMaaS), integrated in accordance with a service-oriented architecture and managed by means of a BPMS that incorporates the new functionality described in the proposal. Subsequently, the implementation of a prototype of this system is provided in conjunction with a test scenario, based on an industrial manufacturing model, which has enabled the viability of the proposal to be validated and 
demonstrated. Finally the main research conclusions are presented in conjunction with future research proposals.

\section{Background}

The full integration of manufacturing processes into the global map of the business model has propitiated diverse solutions that have developed from traditional automation models (Modbus, Profibus, AS-I, FIPIO, DeviceNET, Interbus and Industrial Ethernet) integrated with the management levels by means of ad hoc adapters to systems based on information technology applications that are currently achieving great success within the scope of integration, such as the transparent factory proposal [5], the use of the SOAP protocol as proposed by ABB [6] and systems based on Web Services [7]. There are more recent proposals, some of which are within the scope of European projects based on the use of Web Services and WS-*, that show the functionality of the production elements [8]. Other proposals, such as IMaaS, which is described in [4][9], present industrial machinery as part of a business process management system (BPMS), indicating its functionality as services in accordance with the SOA paradigms. This latter focus is one that has been used in the general scenario described in the proposal of this paper in order to enable the implementation of BPMS in manufacturing organisations. The IMaaS proposal eliminates the aforementioned technological and conceptual restrictions that prevented the integration of business processes and manufacturing processes. IMaaS defines a process that conceptually and technologically normalises industrial machinery. Conceptual Normalisation establishes a model that removes conceptual restrictions between manufacturing process and business processes. The Technological Normalisation of industrial machinery establishes the architecture (IMaaS architecture) that removes former technological restrictions [4].

In order to cover requirements such as selforganisation, automation and reactive and proactive behaviour, it is necessary for there to be communication between the organisation, the applications and the machinery. To enable this communication to exist it is essential to formally classify the information referring to a domain, for which ontologies are presented as useful tools. This information is interpretable by software agents in such a way that the interoperability of IT systems is enabled and the integration of the different systems within the company is strengthened, thus making it possible to establish reasoning, based on the internal logic of the ontology, that allows the different elements to automatically interact. Two major ontology development areas can be distinguished with regard to the domain of manufacturing companies: the conceptualisation of manufacturing domains and the application of semantics to the management of business processes.
The conceptualisation of the domain is a fundamental element within the use of ontologies. There are a number of different papers that list the concepts involved and identify the relationships between them. Based on these studies, we can generate taxonomies that list all the concepts in order to form the ontology. Examples of these studies are [10], where the use of ontologies is proposed within the ADACOR architecture. In [11] another, more specific manufacturing domain ontology is proposed: the MASON (MAnufacturing Semantics ONtology) ontology, based on the description of the domain as a sum of resources, operations and entities, proposed by [12]. Continuing with this theme, in [13] ontology is used as an element to represent knowledge by means of instances of the resources available for the machinery and to achieve, therefore, machinery that is capable of automatically reconfiguring itself in accordance with the objective.

Once the elements involved in the production systems have been identified, use can be made of semantics for the management of processes. There is a series of proposals that are based on semantically representing the services that the machines can offer. In [14], the use of Semantic Web Services for the automation of production systems is put forward. It is based on the hypothesis that if the manufacturing components can be described using semantics interpretable by machines, then the other intelligent components can reason and deduce the knowledge sufficient to reciprocally act. In [15] the use of Semantic Web Services such as Middleware is proposed for the reconfiguration of manufacturing systems. Thanks to the semantic description of the services and to a series of rules that model certain events, the automatic selection of services based on a series of events and objectives is enabled.

However, manufacturing cannot be considered to be an isolated process within the company, rather it must be understood to be just another process within the company's business processes. In this field there is also a wide range of proposals. In [16], an architecture based on the semantic description of services and on a BPEL (Business Process Execution Language) engine is proposed as the means for executing said services. However, there is strong debate with regard to which perspective is the most appropriate for the dynamic composition of services and as yet a consensus does not seem to have been reached. In [17] a comparison is made between BPEL (Business Process Execution Language) and OWL-S (Ontology Web Language for Web Services) and the conclusion is that OWL-S is more appropriate for dynamic environments and BPEL is the ideal choice for controlled workflows. In [18] the replacement of BPEL4WS (Business Process Execution Language for Web Services) technology by Semantic Web Services is proposed. Accordingly, a tool to translate BPEL to OWL-S is proposed. In [19] a set of limitations on BPM methodologies is identified and the 
proposal to overcome them is a combination of BPM and SWS (Semantic Web Services) leading to what the authors have called Semantic Business Process Management.

From the analysis of the above proposals it can be discerned that currently BPM systems are the most appropriate for dynamic management processes and that IMaaS has been presented as the solution to enable the implementation of BPMS in manufacturing organisations as it enables the technological and conceptual barriers that exist between the levels to be overcome. Notwithstanding, current BPM system proposals do not minimise the increase of the process modelling tasks created by the introduction of new production models such as mass customization. In order to minimise the workload of process engineers it is necessary to automate these modelling tasks and thus achieve rapid adaptation to change. As we have seen in this section, ontologies are presented as the most efficient form for equipping a system with a greater level of automation by means of the incorporation of knowledge. Accordingly, this paper proposes the automation of the process model by means of the use of ontologies incorporating this new functionality to BPMS platforms and resolving the aforementioned gaps in said management systems.

\section{Proposed ontology}

As we have seen in the previous section, the use of ontologies in industrial environments is especially appropriate for several reasons: first, ontologies enable the production and business levels domain to be reflected by establishing an explicit relationship between the elements. Accordingly, machinery can interact with the rest of the company's elements independently of the manufacturer and of its technological and functional characteristics. Second, we can establish automatic reasoning series, based on a set of given rules, that enable the different elements of the production system to act autonomously in response to requests from the process manager, thus converting the machinery into an intelligent agent within the production system. Moreover, given that the industrial domain is relatively stable, the conceptualisation of the different elements and the relationships between them is viable by means of the use of formal ontologies.

This proposal is focused on producing a semantic description of the physical structure of manufacturing organisations, the description of the possible processing services and their relationship with the different machines and with other processing services.

These generic definitions can be used to define the instances referring to a particular domain, reflecting the relationships and restrictions between the different instances that facilitate automation when composing new processes. The main classes and relationships of the ontology are explained. First, a brief study of the domain is made in order to subsequently carry out the conceptualisation and implementation of the ontology.

\subsection{Study of the domain}

The study of the domain has been carried out taking into account the classification of processes described in [20]. It identifies three groups of production processes: coordination and control, transport and storage, processing and assembly. In turn, each one is divided into more specific processes, which we have not detailed for reasons of space. Second, an analysis of the main machines used in manufacturing organisations has been made and the relationships between the different processes have been established: for example, transport processes can only be undertaken by conveyor belts, turn tables, rail carriages, etc. and milling machines cannot be used for assembly processes.

Furthermore, in a manufacturing plant the machines do not act independently of each other, rather it is the combination of the activities undertaken by each machine that leads to the finished manufactured product. Accordingly, the way in which the machines co-operate must be defined. In this case, it is mainly due to geographical proximity, transporting materials to adjacent machines or processing them with machines situated on a specific belt.

Finally, it should be stated that manufacturing processes can be composed of sub-processes or have other types of associated processes such as support processes.

\subsection{Conceptualisation}

In this section, the vocabulary and axioms referring to the domain (called TBox in descriptive logic) are defined and conceptualisation is carried out in accordance with the three aspects mentioned above: physical components and the relationship between them; activities, processes and the relationships between them; and the relationships between processes and physical elements.

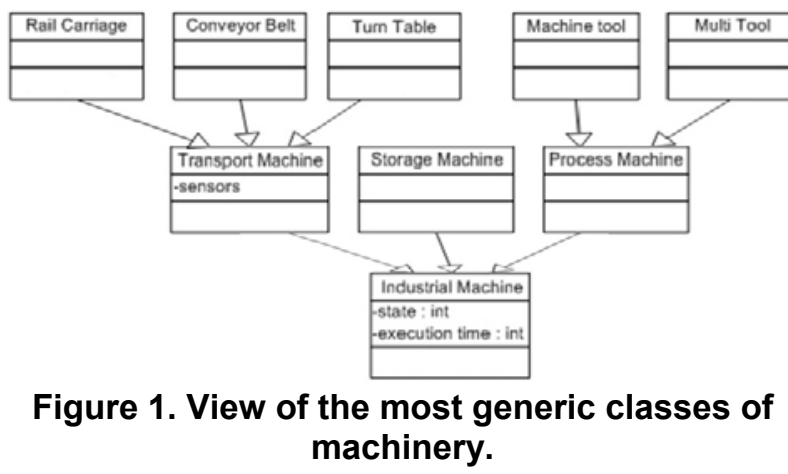

The conceptualisation of the physical elements implies the definition of numerous classes. It should be stated that industrial machinery has been defined as the main class, identifying transport machinery, processing machinery and storage machinery as its subclasses. Each 
subclass, in turn, is composed of others that represent more specific machinery.

For these classes there is a series of logical relationships in accordance with their physical relationships. Some of the relationships are described below in accordance with the position of the machines with respect to other machines (specified in first-order logic). These relationships have special relevance in the reasoning shown in section 5. For example, any Transport Machine (tr1) can be physically connected to another Transport Machine ( $\operatorname{tr} 2)$ on one of its sides of the $\mathrm{X}$ axis (positive and negative $\mathrm{X}$-axis). These relationships are inherited by any of the subclasses of the Transport Machine concept shown in Fig. 1. such as Rail Carriage (RC), Conveyor Belt (CB) and Turn Table (TT).

\section{( $\forall \operatorname{tr} 1, \operatorname{tr} 2)$ hasAxisXPositive(tr1,tr2)}

$(\forall \operatorname{tr} 1, \operatorname{tr} 2)$ hasAxisXNegative $(\operatorname{tr} 1, \operatorname{tr} 2)$

In accordance with the geographical relationships between the different physical elements, a series of rules can be extrapolated to establish which machines are physically connected to each other (hasDirectConnection) and which machines can be reached through other machines (hasConnection). For example, if a Transport Machine (tr1) has a physical connection with another Transport Machine (tr2) at either end (hasAxisXPositive/Negative) then it is directly connected.

$(\forall \operatorname{tr} 1, \operatorname{tr} 2)$ hasAxisXPositive(tr1,tr2) $\mathrm{V}$ hasAxisXNegative $(\operatorname{tr} 1, \operatorname{tr} 2) \rightarrow$ hasDirectConectionOnAxisX $(\operatorname{tr} 1, \operatorname{tr} 2)$

If a Transport Machine ( $\operatorname{tr} 1)$ is physically connected to another $(\operatorname{tr} 2)$ and this in turn is directly connected to a third Transport Machine (tr3), then there is at least one connection between the first machine $(\operatorname{tr} 1)$ and the third machine (tr3), so they are both connected.

$(\forall \operatorname{tr} 1, \operatorname{tr} 2)$ hasDirectConection $(\operatorname{tr} 1, \operatorname{tr} 2) \wedge$ hasDirectConection(tr2,tr3) $\rightarrow$ hasConection $(\operatorname{tr} 1, \operatorname{tr} 3)$

Manufacturing domain processes and activities have also been identified, establishing as the main class the generic concept Process with its subclasses defined as transport, processing and storage. Each one of these classes is divided, in turn, into other subclasses that represent more specific concepts such as in the case of processes: drilling, milling, move to end, move to sensor, etc. This continues until the activity level is reached, where no further division is made, Fig. 2.

The main relationships identified between processes are composition and association. The production process can be composed of several subprocesses or the execution need of a process can be linked to the execution of another process, as is shown below. For example, a manufacturing process $(\mathrm{mp})$ can be a subprocess of a production process (pp).

\section{( $\forall \mathrm{mp}, \mathrm{pp})$ SubProcesOf(mp,pp);}

Finally, the relationships between the production processes and the industrial machinery that can carry out the processes are established in the ontology. The machinery offers these processes as services, in accordance with the IMaaS architecture, so a process may be implemented by more than one machine. The services are classified in accordance with the type of machinery that offers them.

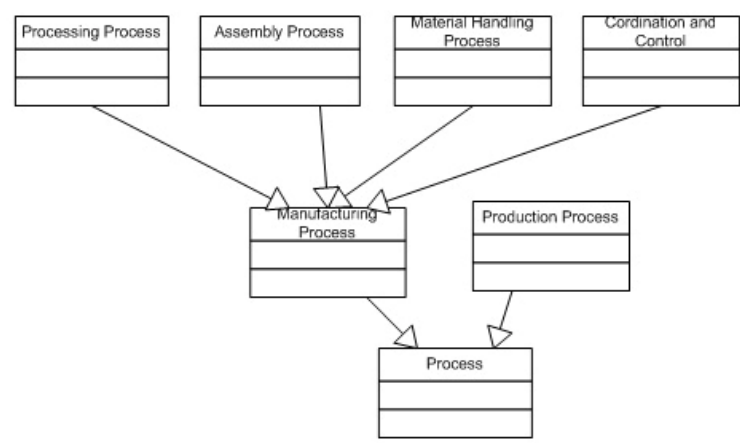

Figure 2. View of the most generic classes of the process classification.

Relationships are established both with the classes that conceptualise the processes and with the conceptualisation of the types of machinery. It is specified which service implements each process and which machinery offers each service. The relationships between the machines and the processes they implement are shown below in addition to the relationships between the services offered and the machines that offer them. For example, a transport service fig. 5 (ts) implements a material handling process fig. 4. (mhp), or a machine tool service (mts) implements a manufacturing process.

( $\forall$ ts,mhp)Implements(ts,mhp)

( $\forall \mathrm{mt}, \mathrm{mp})$ Implements(ms,mp)

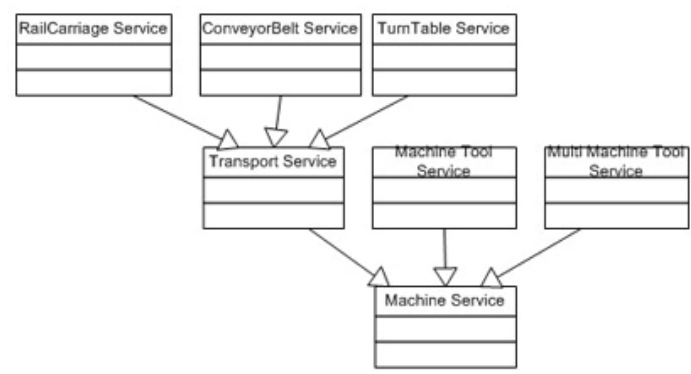

Figure 3. View of the most generic classes of the service classification.

\subsection{Reasoning base}

Thanks to the ontology, a reasoning series can be established to enable the automatic selection of the services required to be able to carry out the processes 
modelled by the process engineer in accordance with the manufacturing objectives. In this way the knowledge required to automatically compose, based on the objectives, a process that can be carried out by a specific industrial plant can be extracted.

By making a simple layout of how this knowledge can be generated we can appreciate the importance of identifying the correct relationships between classes.

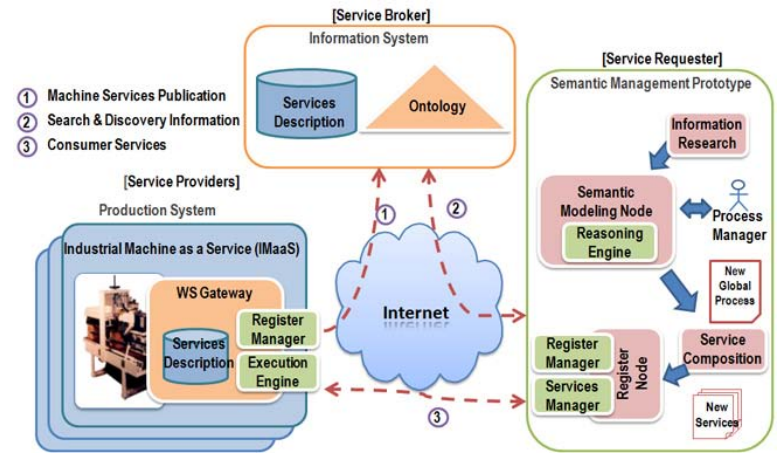

Figure 4. Functional diagram of the proposed system

Accordingly, the semantic modelling node must be initially aware of where material is going to be processed and what processes are going to be carried out. In accordance with these processes, the services that implement processes are identified in addition to the industrial machinery that offers them. Once the machines that are capable of carrying out the process are identified, the machines through which the material must pass in order to be processed are calculated. Depending on each machine, the associated service will be automatically selected in order to implement it and continue on to the adjacent machine until the raw material reaches the machine corresponding to processing. In this way we can identify between which processes there is a dependent relationship, in such a way that the selection of one of them implies that both are selected for the composition of the new process.

\section{General proposal}

The proposed model only makes sense if it can be developed in realistic scenarios that include the needs of both of the business and of the technologies used to sustain it. For this reason, in this paper we have proposed a general scenario based on SOA architecture in which industrial machinery acts as a supplier of services (IMaaS), and the business policies, objectives and strategies are managed by means of a BPMS that acts within this SOA scenario as an IMaaS service consumer. This BPMS (Fig. 4) has been functionally enriched to include the automation proposal in the process model described in this paper. It is these services, which are modelled as processes, that are automated by the proposed dynamic manufacturing model.
The scenario that has been designed to support the dynamic production system is composed of three elements: the production system, the information system and the semantic process management system. The scenario is organised in accordance with the SOA paradigm where the production system takes on the role of service provider; the information system encompasses the service intermediary and the ontology that contains the conceptualisation of the company's business elements; and the semantic process management system represents the role of process modeller and service consumer.

The production system is composed of IMaaS industrial machinery [13] and is responsible for carrying out the modelled processes by means of the services that it offers. These services are registered in the information system as can be seen in the Publication block in Fig. 5 so they can be discovered by the rest of the elements of the company.

The semantic process management system is responsible for managing the new production processes, creating, based on the indications of the process engineer, a complete process that can be undertaken by the production plant, which enables the process engineer to model in accordance with the established policies and desired objectives and with the specific production elements operation mode. The Semantic Modelling Node will be responsible for generating the new production processes based on the semantic information extracted from the instances defined in the proposed ontology. This enables the process engineer to be released from much of his workload. Once the new modelled process is available, it is automatically translated to an execution language, discovering the services offered by the machinery that implements the required subprocesses and sending the new process to the industrial machinery for execution. In the Semantic Management block in Fig. 5 the sequence of activities for semantic process modelling is shown.

In a traditional BPMS process modelling is a tedious activity that often requires the collaboration of several process managers. It is assumed that each one of the process managers has sufficient knowledge of the domain and is fully responsible for the modelling and, therefore, for the suitability of the manufacturing process. Moreover, once the new process has been modelled it is necessary to specify the services that are necessary in order to be able to compose the new service, as can be seen in the BPMS block in Fig. 5 .

Once composed, the new process is registered so that it can be used again in the future.

The information system is responsible for recording all available processes and updating information about the industrial domain in such a way that any change that may affect the functioning of the business will be taken into account when new processes are generated by the semantic process management system. 


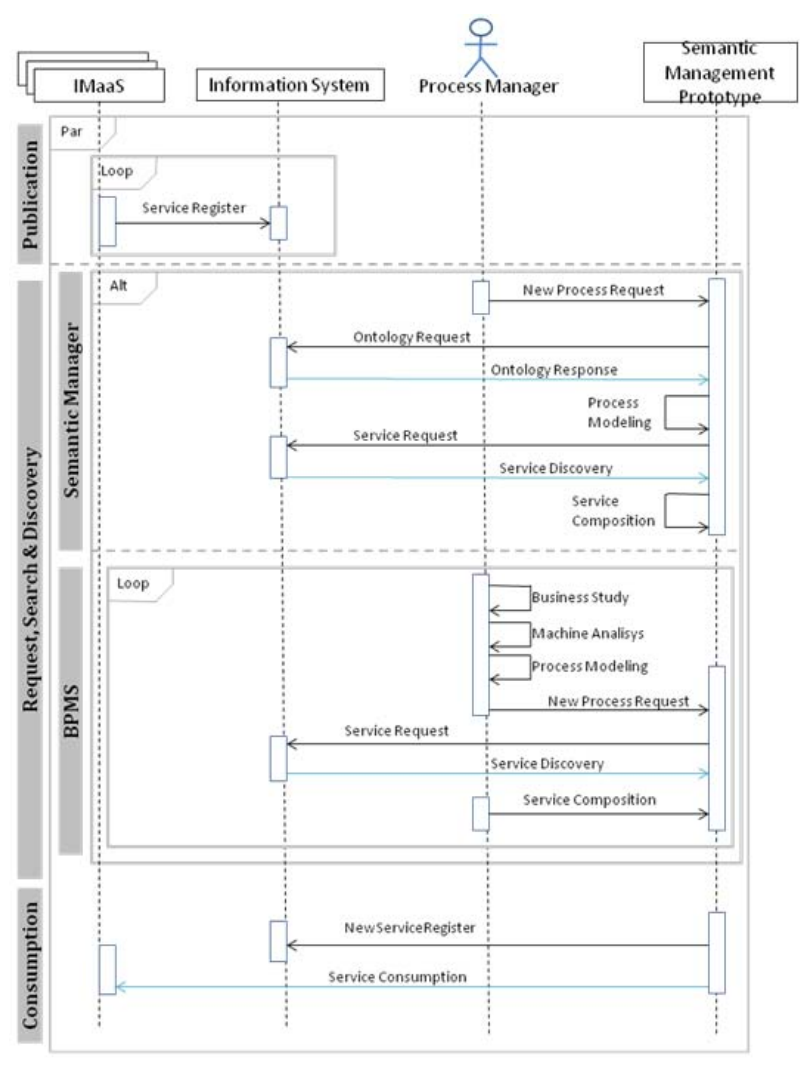

Figure 5. Sequence diagram: creation of a new
process

Thanks to semantic management, information can be extracted from the ontology in order to model the processes, which enables greater automation when composing processes. The system also automates the selection of the services that are carried out. As can be seen in the Request, Search and Discovery block in Fig. 5 , the semantic management system enables a reduction of the required interaction between the business management system and the process manager with respect to a traditional BPMS.

\section{Test and validation scenario}

In order to validate this proposal a global test scenario has been defined. It is based on Fig. 4, in which the validation process has been applied to a scale model that simulates an industrial manufacturing process.

\subsection{Global test scenario}

As we have mentioned in previous sections, the identified problem consists of the difficulties inherent in modelling multiple and complex manufacturing processes. In our proposal (Fig. 4), the semantic management prototype is responsible for this function.

The main objective of this prototype, in addition to the consumption of services, is to mitigate the problems in question. Thanks to the rules of inference and to a semantic reasoning engine, the system is capable of automatically ordering and locating the services necessary to undertake new processes.

The Semantic Management prototype has been implemented with the Jena library to manage the ontology and Bossam as the reasoning engine for obtaining the inferences. The definition of the new process is translated to a BPEL sheet that contains the description and sequencing of the operations to be carried out by the industrial machinery.

The second element of the scenario is the manufacturing system, or service provider. This system is composed of a scale model of an industrial factory and its design can be seen in Fig. 6 and Fig. 7 shows a photo of the same. This production system, which was designed by the information technology and computing department of the University of Alicante, has been implemented by the company STAUDINGER GMBH, and is composed of the following industrial machines: a flexible production line formed by a milling machine, a drilling machine and pneumatic drill, a manufacturing cell with a milling machine and several conveyor belts. Communication with each element of the model is carried out by means of an RS-485 interface using the DCOM industrial protocol. The service provider is implemented in the Lantronix brand generic incorporated device called XPORT, whose characteristics are suitable for the preparation of the platform, such as its very small size and good computational capacity.

Table 1 shows the definition of the process map of the industrial machines that compose the scale model. In the description of these services, for reasons of space, a nomenclature based on the process inheritance between the different components has been followed, in such a way that newComponent:BaseComponent means that the element defined in NewComponent includes all the processes defined in BaseComponent.

The information system encompasses all the elements and resources used by the rest of the systems within the organisation in order to carry out the business or company activities. These elements include the UDDI (Universal Description, Discovery and Integration) record, implemented with the jUDDI server that stores the WSDL (Web Service Description Language) and BPEL documents that describe the functionalities and business processes of the organisation and the ontology with the information referring to the conceptualisation of the specific scenario, published directly on the APACHE TOMCAT server.

5.2. Implementation of the ontology and instantiation

The implementation of the ontology has been carried out by means of the OWL DL (Ontology Web Language Description Logic) language. This language has been chosen in order to represent the ontologies because it is the language that enables the greatest level of expression while maintaining the principles of completeness and computational decidability. In conjunction with this 
language, the SWRL (Semantic Web Rule Language) rules language has been used to model certain relationships of the domain concepts.

\begin{tabular}{|c|c|c|}
\hline Name & Arguments & Description \\
\hline \multicolumn{3}{|c|}{ Conveyor Belt (CB) } \\
\hline $\begin{array}{l}\text { MoveTo } \\
\text { Sensor }\end{array}$ & $\begin{array}{l}\text { Direction:INT }\{1,-1\} \\
\text { Sensor:INT }\{0-\mathrm{N}\} \\
\text { Stop:BOOL } \\
\end{array}$ & $\begin{array}{l}\text { Move the piece to } \\
\text { until indicated } \\
\text { sensor. }\end{array}$ \\
\hline MoveToEnd & Direction:INT $\{1,-1\}$ & $\begin{array}{l}\text { Move the piece to } \\
\text { the end of the belt. }\end{array}$ \\
\hline \multicolumn{3}{|c|}{ Turntable:Conveyor Belt (TT) } \\
\hline TurnMove & Direction:INT $\{1,-1\}$ & $\begin{array}{l}\text { Turn the table and } \\
\text { expedite the piece }\end{array}$ \\
\hline \multicolumn{3}{|c|}{ Rail Carriage:Conveyor Belt (RC) } \\
\hline Transport & $\begin{array}{l}\text { Direction:INT }\{1,-1\} \\
\text { InputDirection:INT } \\
\text { OutputDirection:INT }\end{array}$ & $\begin{array}{l}\text { Get a piece, } \\
\text { transport and } \\
\text { expedite it. }\end{array}$ \\
\hline \multicolumn{3}{|c|}{ Machine Tool (MT) } \\
\hline Process & Time:INT & $\begin{array}{l}\text { Move the tool out } \\
\text { and down, process } \\
\text { the piece and } \\
\text { move the tool up } \\
\text { and in. }\end{array}$ \\
\hline \multicolumn{3}{|c|}{ Machine Multi Tool:Machine Tool (MMT) } \\
\hline $\begin{array}{l}\text { ProcessWith } \\
\text { Tool }\end{array}$ & $\begin{array}{l}\text { Tool:INT }\{0-2\} \\
\text { Time:INT }\end{array}$ & $\begin{array}{l}\text { Process the piece } \\
\text { with the indicated } \\
\text { tool. }\end{array}$ \\
\hline
\end{tabular}

\section{Table 1. Services offered by the industrial} machinery.

The instances have been defined in accordance with the elements available in the industrial scale model (Fig. 6) and the services they offer, modeling nine Conveyor Belts, four Turn Tables, one Rail Carriage, three Machine Tools, and one Multi-Tool machine. In order to implement the ontology and the definition of the initial instances, the Protégé tool has been used, resulting in the OWL document that has been published as part of the information system of the proposal.

\subsection{Experimentation}

In order to validate the proposed model, a series of tests has been carried out to demonstrate the use of the model for the composition of manufacturing processes in accordance with the reasoning of the information available in the ontology.

Accordingly, the system was informed of a series of processes to be undertaken and the initial location of the raw material. Based on this, the system was able to correctly identify the machines necessary for processing the material in accordance with the request and the shortest routes were calculated (in this case the paths crossing the least number of machines) and, finally, the services associated with the machines were selected in order to move the goods to the machine that would process them.

The same test was carried out with one of the transport machines disconnected. The system was able at all times to select the most suitable machinery in order to transfer the material to the machine that had to process it.

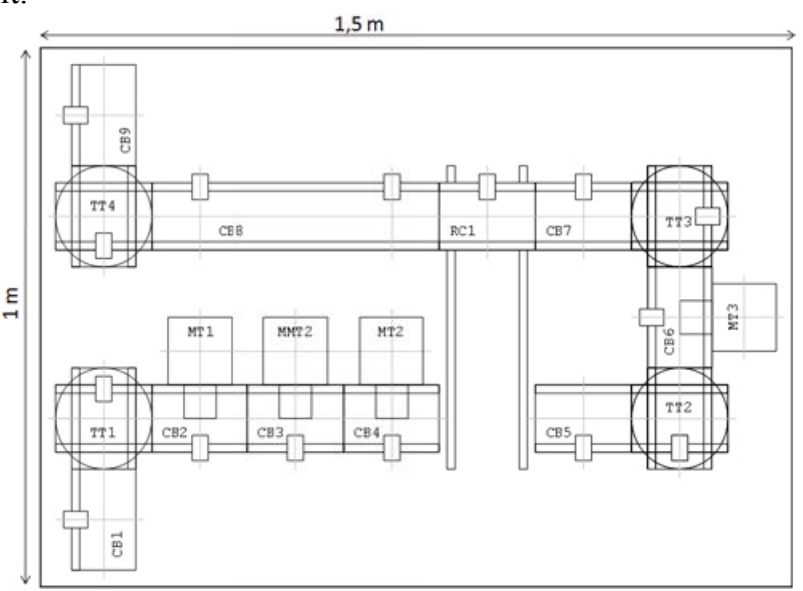

Figure 6. The manufacturing model

A machine that carried out one of the selected processes was also disconnected. Also in this case, the semantic management prototype was able to find another machine that was capable of carrying out this process and recalculated the path required to reach this machine, correctly selecting the services available.

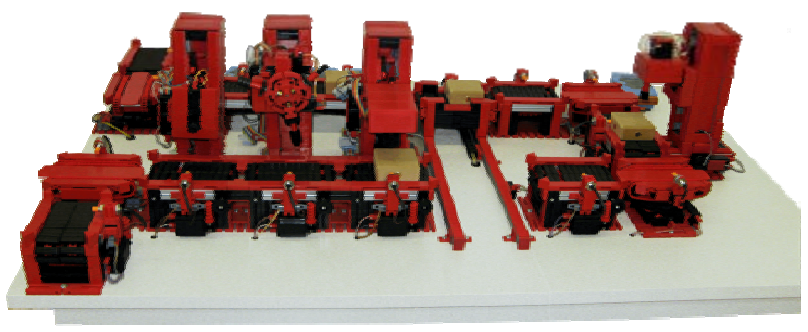

Figure 7. Industrial model for testing.

\section{Conclusion}

This paper has proposed an automated manufacturing process management model based on the use of ontologies. Its objective is to mitigate the process modeling workload of process engineers, automatically inferring, in accordance with the semantic relationships of the components of the industrial domain, the processes and activities required to compose a new process.

Furthermore, we have proposed a general scenario based on SOA architecture in which industrial machinery behaves as a service provider and the business policies, objectives and strategies are managed by a BPMS which operates within this SOA scenario as an IMaaS service consumer with the incorporation of the 
functionality described in the proposal. The modelling of the services, contemplated as processes, is automatically managed by the inference engine incorporated into the proposed dynamic manufacturing model whose functionality has been added to the BPMS.

Although some major questions still have to be resolved, the implementation of the model and of the proposed scenario has enabled the viability and utility of the proposal to be verified. It has been verified that automatically modeled processes have been satisfactorily adapted to the plant instanced in the ontology.

Currently, work is under way to modify the proposed semantic management system in order to contemplate the composition of processes by means of BPMN (Business Process Management Notation) annotation. We are also developing a system that will enable the automatic generation of semantic descriptions of the services offered in OWL-S. This will enable us to achieve a higher level of automation in the composition of new processes in accordance with the company's objectives and policies.

\section{Acknowledgements}

This work was supported by the Spanish Ministry of Education and Science with Grant TIN2006-04081.

\section{References}

[1] C. Younghwan, K. Kwangsoo, K. Cheolhan. "A design chain collaboration framework using reference models". Innternational Journal of Advanced Manufacturing Technology. 26 (1) pp. 183-190. July, 2005.

[2] H. Smith and P. Fingar. "Business Process Management. The Third Wave". Meghan-Kiffer, 2002.

[3] J.F. Chang. "Business Process Management Systems. Strategy \& Implementation”. Auerbach Publications'05.

[4] V. Gilart-Iglesias, F. Maciá-Pérez, D. Marcos-Jorquera and F. J.Mora-Gimeno. "Industrial Machines as a Service: Modelling industrial machinery processes". Proc. of 5th International IEEE Conference on Industrial Informatics (INDIN'07).Vienna, 2007.

[5] Transparent Factory. Manual de usuario y planificación. [Online] Available: http://www.modicon.com, 2001.

[6] U. Topp, P. Müller. "Web based service for embedded devices". International Workshop on Web Service: Research, Standardization and Deployment (WSRSD'02). Lecture notes in computer science.

[7] A.P. Kalogeras, J.V. Gialelis, C.E. Alexakos, M.J. Georgoudakis, S.A. Koubias. "Vertical integration of enterprise industrial systems utilizing Web Service," Proc. of the 5th IEEE International Workshop on Factory Communication System (WFCS 2004), Technical University of Vienna, Vienna, Austria, 22-24 Sep. 2004.

[8] F. Jammes, H. Smit. "Service-Oriented paradigms in industrial automation," IEEE Transaction on industrial informatics. VOL I. $n^{\circ}$ 1, pp. 62-70. 2005.
[9] V. Gilart-Iglesias, F. Maciá-Pérez, J.A. Gil-MartínezAbarca and A. Capella-D'alton. "Industrial Machines as a Service: A model based on embedded devices and Web Services". Proc. of 4th International IEEE Conference on Industrial Informatics (INDIN'06). Singapore, 2006.

[10] Borgo, S., Leitão, P., "Foundations for a Core Ontology of Manufacturing", Ontologies. A Handbook of Principles, Concepts and Applications in Information Systems vol. 14, pp 751-775, Springer, 2007

[11] A. Siadat, J. Y. Dantan, A. Semenenko, S. Lemaignan. "MASON: A proposal for ontology for Manufacturing Domain". Proc. of the IEEE Workshop on Distributed Intelligent Systems: Collective Intelligence and Its Applications (DIS'06) 2006.

[12] P. Martin and A. D'Acunto. "Design of a production system an application of integration product-process". Int. J. Computer Integrated Manufacturing, 16(7-8):509516, 2003

[13] Y. Al-Safi, Valeriy Vyatkin. "An Ontology-Based Reconfiguration Agent for Intelligent Mechatronic Systems". Proc. of the 3rd international conference on Industrial Applications of Holonic and Multi-Agent Systems: Holonic and Multi-Agent Systems for Manufacturing. LNAI 4659, pp. 114-126, 2007. Springer-Verlag Berlin Heidelberg 2007

[14] J. L. Martinez-Lastra, I. M. Delamer. "Semantic Web Services in Factory Automation: Fundamental Insight and Research Roadmap" .IEEE Transactions On Industrial Informatics. vol 2. $\mathrm{n}^{\circ} 1$. feb 2006.

[15] I. M. Delamer. "Event-based Middleware for Reconfigurable Manufacturing Systems: A Semantic Web Services Approach". Doctoral thesis. Tampere University of technology. 2006.

[16] Y. Yamato, Y. Nakano, H. Sunaga. "Study and Evaluation of Context-Aware Service Composition and Change-Over Using BPEL Engine and Semantic Web Techniques." Proc. of the Fifth IEEE Consumer Communications \& Networking Conference”. (CCNC) 2008.IEEE Computer Society.

[17] W. Ren, G. Chen, D. Chen, C. Ping-Low, C. Sun, J. Bing-Zhang, Z. Yang . "Search for Service-Oriented Strategies of Dynamic Composition of Web Services: A Comparative Perspective". 33rd Annual Conference of the IEEE Industrial Electronics Society. 2007

[18] J. Shen, Y. Yang, C. Zhu, C. Wan . "From BPEL4WS to OWL-S: Integrating E-Business Process Descriptions". Proc. of the 2005 IEEE International Conference on Services Computing. vol 1, pp $181-190$.

[19] C. Pedrinaci, C. Brelage, T. Lessen, J. Domingue, D. Karastoyanova, F. Leymann. "Semantic Business Process Management: Scaling up the Management of Business Processes". 2nd IEEE International Conference on Semantic Computing (ICSC) 2008, IEEE Comp. Society

[20] M.P. Groover. "Automation, Production Systems, and Computer-Integrated Manufacturing". Prentice Hall. 2001. Web, Web Service and Database Systems, pp. 141$153,2002$. 